\title{
VLSI Circuit Configuration Using Satisfiability Logic in Hopfield Network
}

\author{
Mohd Asyraf Mansor \\ School of Mathematical Sciences, Universiti Sains Malaysia, 11800, Pulau Pinang, Malaysia \\ E-mail: asyrafalvez@live.com \\ Mohd Share duwan M. Kasihmuddin \\ School of Mathematical Sciences, Universiti Sains Malaysia, 11800 USM, Penang Malaysia \\ E-mail: iwanmaidin@gmail.com \\ Saratha Sathasivam \\ School of Mathematical Sciences, Universiti Sains Malaysia, 11800 USM, Penang Malaysia \\ E-mail: saratha@usm.my
}

\begin{abstract}
Very large scale integration (VLSI) circuit comprises of integrated circuit (IC) with transistors in a single chip, widely used in many sophisticated electronic devices. In our paper, we proposed VLSI circuit design by implementing satisfiability problem in Hopfield neural network as circuit verification technique. We restrict our logic construction to 2-Satisfiability (2-SAT) and 3Satisfiability (3-SAT) clauses in order to suit with the transistor configuration in VLSI circuit. In addition, we developed VLSI circu it based on Hopfield neural network in order to detect any possible error earlier than the manual circuit design. Mic rosoft Visual $\mathrm{C}++2013$ is used as a platform for training, testing and validating of our proposed design. Hence, the performance of our proposed technique evaluated based on global VLSI configuration, circuit accuracy and the runtime. It has been observed that the VLSI circuits (HNN-2SAT and HNN-3SAT circuit) developed by proposed design are better than the conventional circu it due to the early error detection in our circuit.
\end{abstract}

Index Terms-VLSI circuit, Hopfield Network, 2Satisfiability, 3-Satis fiab ility, Circu it accuracy, Transistor.

\section{INTRODUCTION}

Very Large Scale Integration (VLSI) is a process of creating a circuit with thousands of transistors in a single chip. VLSI had been implemented in numerous application in electronic circuit (EC) such as CPU, RAM and ROM. The concept of VLSI helps the integrated circuit designer to combine thousands of transistors in a single chip. Structured VLSI design were coined by Carver Mead and Lynn Conway in 1980. VLSI structure consists of repetitive arrangement of rectangular macro blocks which are connected by background wire [30]. This blocks consist of discrete bits and each bit will contribute to VLSI function. According to Moore's law, the number of transistor per chip will be double in each technology generation [31]. The complexity of the VLSI design will increase as the number of electronic components increase. This design lead to rapid development of optimal VLSI design. Moreover, the discrete nature of VLSI design makes it became popular in the combinatorial field.

Traditionally, late error detection in the VLSI design phase is a major cause of unexpected delays in the real circuit hardware $[6,12]$. This problem inspires researchers to create model verification techniques which help in earlier error detections. For many practical reasons, it is becoming increasing difficult to manually build a functional VLSI circuit. Modern VLSI usually integrated with static complementary logic gate structures $[13,16]$. Logic gate design in VLSI structure is a methodology that drives an output to either high or low. Neural network hold considerable promise in rapid model creation of VLSI design [4, 11]. By assuming binary input as signals, Boolean logic can be used to represent logic construct in VLSI design. Asynchronous circuits are fundamental in many high performance digital computer systems $[6,12]$. Due to its importance in vast applications, the features of asynchronous circuits cannot be ignored. Asynchronous design is proven to be extremely complex and most cases can be formulated as satisfiability problem [10, 14]. Method to solve satisfiability problem play an important role in computing theory and system.

Recurrent neural network are essentially dynamical systems that feedback signals to themselves. Popularized by John Hopfield, these models possess a dynamical system with stable states with each own basin and attraction [17]. Hopfield neural network minimizes Lyapunov energy due to physical spin of the neuron states. Content addressable memory feature in Hopfield network is proven to be very useful to solve combinatorial optimization problem $[3,24]$. The network produced optimal output by minimizing the network energy. Gadi Pinkas and Wan Abdullah [2, 5] defined a bi-directional mapping between logic and energy function of symmetric neural network. To frame their novelty, 
both method are applicable to validate the solution obtained are models for a corresponding logic program. The work of Sathasivam [2] showed that the optimized recurrent Hopfield network could be possibly used to do logic programming. Intelligent way of defining connection strength of the logic helps the network to suppress spurious state $[1,5]$. In this paper, we proposed Hopfield network to model circuitSAT. The main idea of these simulations is to demonstrate the computational power of collective HNN-kSAT in modelling optimized VLSI circuit.

This paper has been organized as follows. In section II, the fundamental theory of satisfiability problems, namely 2-Satisfiability (2-SAT) and 3-Satisfiability are discussed. Section III emphasizes the concept of VLSI circuit satisfiability, including the fundamental concept of Boolean circuit and the VLSI reconstruction. Furthermore, section IV revolves around the Hopfield neural network and logic programming. Meanwhile, in section V, theory implementation of the networks are been discussed. Finally, section VI and VII enclose the experimental results and conclusion.

\section{SATISFIABILITY (SAT) PROBLEM}

Satisfiability (SAT) is an elementary problem in computer science and mathematics [22]. In VLSI circuits, the transistor problem works similar way as the satisfiability problem. Hence, in order to check the functionality of the VLSI circuit, we opted to integrate the satis fiability problem.

Strictly speaking, a Boolean formula is satisfiable if there exists an assignment of values true and false that renders the entire expression true [27, 29]. The most popular method is exhaustive search, where SAT will try out every possible truth assignment. For instance, given a problem size $n$, there will be $2^{n}$ such assignments and $l$ literals to set for each assignment $[1,20]$. Hence, this method involves $O\left(l .2^{n}\right)$ operations [24]. Hence in general, SAT is an NP-complete problem.

The satisfiability problem concerns Boolean variables or expressions in conjunctive normal form (CNF). CNF comprises of conjunction of clauses, where a clauses are disjunction of literal [3]. Literal is a variable or its negation. For instance:

$$
\left(x_{1} \vee x_{2}\right) \wedge\left(\neg x_{2} \vee x_{3} \vee \neg x_{5}\right) \wedge\left(\neg x_{1} \vee x_{4}\right)
$$

Here $x_{1}, x_{2}, x_{3}, x_{4}$ are Boolean variables to be assigned, $\neg$ means negations (logical NOT), $\vee$ means negations (logical OR), $\wedge$ means negations (logical AND). According to formula in 1 , it will be satisfied when $x_{1}=$ true, $x_{2}=$ false, $x_{3}=$ false, $x_{4}=$ true it takes on the value of true. However, if a formula is not satisfiable, it is termed unsatisfiable, that means that it takes on the value false on any value of its variable.

In VLSI problem, we integrate the core concept of satisfiability in order to obtain correct circuit. The literals in every clauses will be represented by using the transistor. Hence, the ability for the transistor to generate $5 \mathrm{~V}$ is vital in the circuit functionality.

\section{A. 2-SAT}

2-SAT can be defined as a conundrum of determining satisfiability of sets of clauses with at most two literals per clause (2-CNF formulas) [29]. Besides, it is an exceptional case of general Boolean satisfiability which can involve constraints on two variables [19, 22]. In addition, the variables can allow two possibilities for the value of each variable. 2-SAT problem can be expressed as 2-CNF (2-Conjunctive Normal Form). Randomized 2SAT problem is considered as NP problem or nondeterministic problem [28]. The three fundamental components of 2-SAT are summarized as follows:

1. A set of $m$ variables, $x_{1}, x_{2}, \ldots \ldots ., x_{m}$

2. A set of literals. A literal is a variable or a negation of a variable.

3. A set of $n$ distinct clauses: $C_{1}, C_{2} \ldots \ldots . . C_{n}$. Each clause consists of only literals combined by just logical OR $(\vee)$. Each clause must consist of 2 variables.

The Boolean values are $\{1,-1\}$. Researchers have replaced $\mathrm{F}$ and $\mathrm{T}$ in the neural networks by 1 and -1 , respectively to emphasized false and true [13, 29]. Since each variable can take only two values, a statement with $n$ variables requires a table with $2^{n}$ rows. The goal of the 2-SAT problem is to determine whether there exits an assignment of truth values to variables that makes the following Boolean formula $P$ satisfiable.

$$
P=\wedge_{i=1}^{n} C_{i}
$$

Where $\wedge$ is a logical AND connector. $C_{i}$ is a clausal form of DNF with 2 variables. Each clause in 2 SAT has the following form

$$
C_{i}=\stackrel{n}{\vee} \underset{i=1}{\vee}\left(x_{i}, y_{i}\right)
$$

$x_{i} \in\left\{k_{i}, \neg k_{i}\right\}$ and $y_{i} \in\left\{r_{i}, \neg r_{i}\right\} \neg k_{i}$ and $\neg r_{i}$ are negations of the literals.

\section{B. 3-SAT}

In this paper, we emphasize a paradigmatic NPcomplete problem namely 3-Satsifiability (3-SAT). Generally speaking, 3-SAT can be defined as a formula in conjunctive normal form where each clause is limited to at most or strictly three literals $[22,26]$. The proble $m$ is an example of non-deterministic problem [28]. In our analysis, the following 3-SAT logic program which consists of 3 clauses and 3 literals will be used. For instance:

I.J. Intelligent Systems and Applications, 2016, 9, 22-29 


$$
P=(A \vee B \vee \bar{C}) \wedge(\bar{A} \vee \bar{B} \vee C) \wedge(\bar{A} \vee B \vee D)
$$

We represent the above $3 \mathrm{CNF}$ formula with $\mathrm{P}$. Thus, the formula can be in any combination as the number of atoms can be varied except for the literals that are strictly equal to 3. Hence, it is vital for combinatorial optimization problem. The higher number of literal in each clause will increase the possibilities or chances for a clause to be satisfied [29].

The general formula of 3-SAT for conjunctive normal form $(\mathrm{CNF})$ :

$$
P=\bigwedge_{i=1}^{n} Z_{i}
$$

So, the value of $\mathrm{k}$ denotes the number of satisfiability [19]. In our case, k-SAT is 3-SAT.

$$
Z_{i}=\wedge_{j=1}^{k}\left(x_{i j}, y_{i j}, z_{i j}\right), k>3
$$

\section{VLSI CIRCUIT SATISFIABILITY}

VLSI circu it satis fiability is a brand new research area, producing a prolific amount of research. The VLSI circuit design is inspired by the Boolean circuit concept and the other optimization algorithms. Sethuram \& Parashar (2008) has developed the Ant Colony Optimization approach to represent the digital VLSI circuits [32]. On the other hand, some researchers had ventured to model the VLSI circuit by using conventional approach, which is by converting the circuit information into Conjunctive Normal Form (CNF) instances. However, the method results in the loss of the circuit structural configuration especially if the number of the circuit components is getting higher. As a result, we proposed a VLSI circuit based on Boolean circuit by taking into consideration the 3-SAT instances. The integration of 3-SAT instances are able to solve large SAT problems with more of clauses and variables (transistors) by utilizing any advanced intelligence network. Hence, we developed a hybrid model by using Hopfield neural network, logic programming and 3-SAT to configure the input transistor in the VLSI circuit.

\section{A. Boolean Circuit}

A Boolean circuit is a mathematical model for actual logic circu it. Boolean circuit are defined in terms of logic gates which define them. The establishment of the Boolean circuit can be represent in binary input which contain operator such as AND, OR and NOT gates [10, 12]. Each gate corresponds to Boolean function that takes an input bit string and translates it into output bit string [7, $11]$.

Given a finite set $V$ of Boolean variables, $S$ over $V$ is a set of equation of the form $V=f\left(v_{1}, v_{2} \ldots . . v_{k}\right)$ where $v_{1}, v_{2}, \ldots . . v_{k} \in V$ and $f$ is an arbitrary Boolean function.
The variables of $S$ corresponds to the gates of the circuit. A variable can be in the form of "True" or "False".

A truth valuation of $S$ is a function $\Gamma: V \rightarrow\{$ True,False $\}$.Valuation is consistent if $\Gamma(V)=f\left(\Gamma\left(v_{1}\right), \Gamma\left(v_{2}\right) \ldots \ldots\left(v_{k}\right)\right)$ hold for each equation $V=f\left(v_{1}, v_{2} \ldots ., v_{k}\right)$ in $S$. A system $S$ is functional if there exist a consistent valuation for it. The question of whether a system is satisfiable or not, can be considered as NP-complete problem [14]. Note that Boolean Circuit has exactly $2^{n}$ consistent truth assignments, where $n$ is the number of input gates in the circuit [9, 13].

Therefore in case of modelling Boolean circuit, we are interested in the constrained satisfaction problem [11]. Given that the variable in $C^{+} \subseteq V$ must be true and those $C^{-} \subseteq V$ false which is the constraint of the circuit. Figure 1 shows how logic gate in Boolean circuit can be visualize in Quad 2 component.

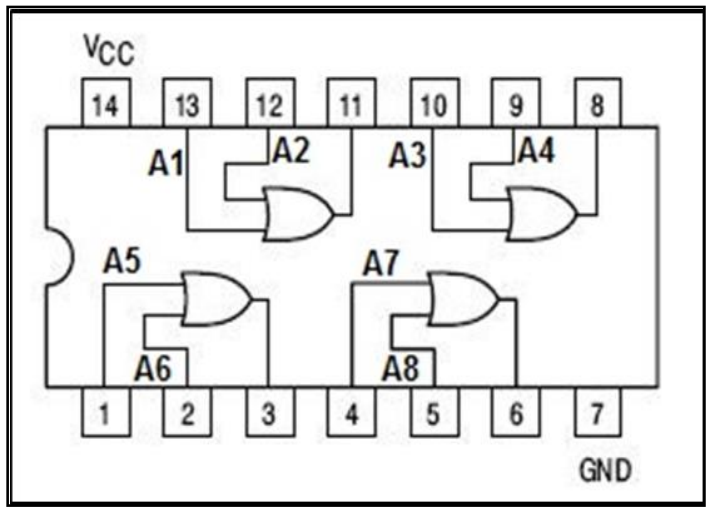

Fig.1. Schematic Diagram of SN74LS32N Quad 2-Input OR Gate

Finding a correct design model circuit is NP problem. The output of the circuit can be physically determined by using any electronics device such as light bulb or ammeter. If the output of the circuit is 1 the bulb will light up or ammeter will show deflection.

\section{B. VLSI Reconstruction}

In essence, Very Large Scale Integration (VLSI) is a process of combining thousands of transistors into a single chip [33]. Transistor is a semiconductor device used to amplify or act as an automatic switch in integrated circuits $[6,9]$. It is composed of semiconductor material with at least three terminals for connection to an external circuit [17, 35]. Transistor consists of base, collector and emitter. In this case, we only consider npn transistor since npn transistor always give us forward bias current. Figure 3 shows a simple diagram of npn transistor.

Modelling a correct VLSI circuit can be treated as combinatorial problem [16]. We are required to find a functional VLSI design model. Figure 2 shows an example of a single unit of a transistor in integrated circuit. 


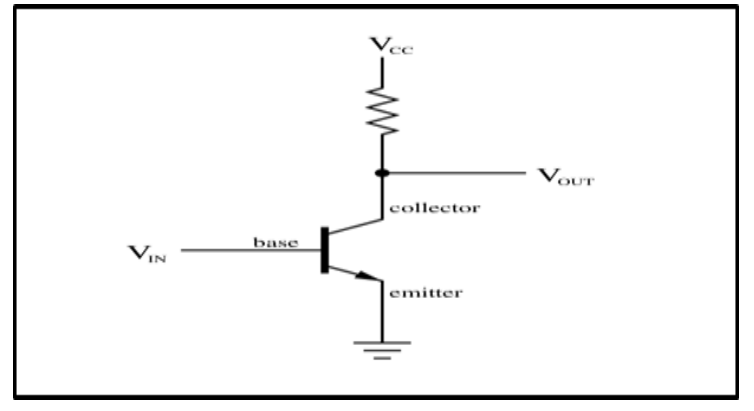

Fig.2. npn-Transistor

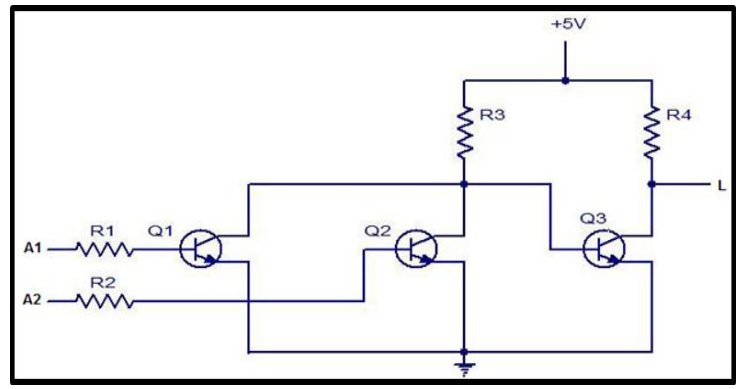

Fig.3. The Input Transistor OR gate

Based on the figure 3, when the inputs $A 1=0 \mathrm{~V}$ and $\mathrm{A} 2$ $=0 \mathrm{~V}$ both the transistors $\mathrm{Q} 1$ and $\mathrm{Q} 2$ are at OFF state (-1). At the same time, transistor Q3 received enough base drive from the supply $+5 \mathrm{~V}$ through resistor $\mathrm{R} 3$. Transistor Q3 will be turned ON. The output of the system is given $\mathrm{L}=\mathrm{V}_{c e}$ corresponds to $0 \mathrm{~V}(-1)$. When either the inputs $\mathrm{A} 1$ and $\mathrm{A} 2$ or both the inputs are equal to $+5 \mathrm{~V}$ (1), then the corresponding transistors either Q1 or Q2 will be $\mathrm{ON}$ (1) or both the transistors Q1 and Q2 will be ON (1). The voltage at the collector of transistor $\mathrm{Q} 1$ is $\mathrm{L}=\mathrm{V}_{c e}$ which corresponds to $0 \mathrm{~V}(-1)$. During this phase, transistor Q3 doesn't forward bias and the base-emitter junction and turns OFF (-1) (No voltage drop across it). Hence the final output voltage corresponds to $+5 \mathrm{~V}$ (logic state 1$)$.

\section{NEURO-LOGIC IN HOPFIELD NEURAL NETWORK}

Neuro-log ic is the combination of neural network and logic programming as a single hybrid network. Basically, the neuro-logic approaches can be applied in a vast fields such as combinatorial optimization, pattern recognition and circuit reconstruction. In this paper, we implement the Hopfield neural network and logic programming to configure the information needed for a VLSI circuit based on 3-SAT instances. The core impetus of this neuro-logic paradig $\mathrm{m}$ is to configure the VLSI circuit in a better way. It can be a robust technique in detecting the early fault in the VLSI circuit. Thus, it will assist us in designing a VLSI circuit with higher complexity. Thus, we can implement the proposed technique in the construction of VLSI circuits for sophisticated electrical device. The effectiveness of neuro-logic paradigm in configuring the VLSI circuit will be discussed in Section VI.

\section{A. Hopfield Model}

In VLSI reconfiguration, we choose the Hopfield neural network because it is distributed and easy to implement. The ability for the transistor in VLSI model to function properly is vital in getting the correct circuit. In our study, we integrate the 2-SAT and 3-SAT problem with Hopfield network in order to relate it with our VLSI reconfiguration conundrum. The correct circuit will be based on the functionality of the transistor. Generally speaking, the Hopfield neural network is an ordinary model for content addressable me mory (CAM) [24]. This features imitates our biological brain whereby learning and retrieving data are the building block of content addressable memory (CAM) [2, 3]. In our case, the VLSI model will be having the possible satisfied transistor combination in order to get the correct circuit.

Strictly speaking, Hopfield neural network is a class of recurrent auto-as sociative network [17, 34]. The units in Hopfield models are principally binary threshold unit [27]. Hence, the Hopfield nets will take a binary value such as 1 and -1 . The definition for unit I's activation, $a_{i}$ are given as follows:

$$
a_{i}=\left\{\begin{array}{l}
1 \text { if } \sum_{j} W_{i j} S_{j}>\xi_{i} \\
-1 \text { Otherwise }
\end{array}\right.
$$

Where $W_{i j}$ is the connection strength from unit $j$ to $i$. $S_{j}$ is the state of unit $j$ and $\xi_{i}$ is the threshold of unit $i$. The connection in Hopfield net typically has no connection with itself $W_{i i}=0$ and connections are symmetric or bidirectional $[4,24]$.

Hopfield network work asynchronously with each neuron updating their state determin istically. The system consists of $N$ formal neurons, each is described by an Ising variable. Neurons are bipolar $S_{i} \in\{1,-1\}$ obeying the dynamics $S_{i} \rightarrow \operatorname{sgn}\left(h_{i}\right)$ where the local field $h_{i}$. The connection model can be generalized to include higher order connection. This modifies the field to

$$
h_{i}=\sum_{j} W_{i j}^{(2)} S_{j}+J_{i}^{(1)}
$$

The weight in Hopfield network is constantly symmetrical. The weight in Hopfield network refers to the connection strength between the neurons. The updating rule maintains as follows:

$$
S_{i}(t+1)=\operatorname{sgn}\left[h_{i}(t)\right]
$$

This properties guarantee the energy will decrease monotonically even though following the activation system [23]. Hence, it will drive the network to hunt for the minimum energy. The following equation represents energy for Hopfield network. 


$$
E=\ldots-\frac{1}{2} \sum_{i} \sum_{j} W_{i j}^{(2)} S_{i} S_{j}-\sum_{i} W_{i}^{(1)} S_{j}
$$

\section{B. Logic Programming in Hopfield Network}

Fundamentally, logic programming can be treated as a problem in combinatorial optimization standpoint [22]. Therefore, it can be carried out in a neural network to obtain desired solutions. Logic programming is user writable and readable [29].

This can be done by using the neurons to store the truth values of the literal and writing a cost function which is minimized when all clauses are satisfied [2, 18]. In other words, the main task is to find 'models' corresponding to the given logic program. The fundamental of Hopfield network in doing logic programming was brought up because of its unique content addressable memory properties.

Implementation of HNN-2SAT and HNN-3SAT in Logic Programming.

i. The 2-SAT and 3-SAT clauses are translated and transformed into Boolean algebra. Basically, the clauses will form a formula that will determine the overall satisfiability. In VLSI circuits, the clauses are referring to the transistor combination in order to generate $5 \mathrm{~V}$ output.

ii. Identify a neuron to each ground neuron.

iii. Initialize all connection strengths or weights to zero.

iv. Derive a cost function that is related with negation of all 2-SAT and 3-SAT clauses. For instance, $X=\frac{1}{2}\left(1+S_{X}\right)$ and $\bar{X}=\frac{1}{2}\left(1-S_{X}\right) . S_{X}=1$ (True) and $S_{X}=-1$ (False). Multiplication represents conjunction and addition represents disjunction.

v. Comparing the cost function with energy, $E$ by obtaining the values of the connection strengths. (Sathasivam's Method) [2, 24]

vi. Check clauses satisfaction by using exhaustive search. Hence, the satisfied clauses will be stored. In VLSI circuits, the satisfied assignments for transistors will be stored as content addressable memory (CAM).

vii. The states of the neurons are randomized. The network undergo sequences of network relaxation [24]. Compute the corres ponding local field $h_{i}(t)$ of the state. If the final state is stable for 5 runs, we ponderit as final state.

viii. Find the corresponding final energy $E$ of the final state by using Lypunov equation. Validate whether the final energy obtained is a global minimum energy or local minima. In VLSI circuit, the final energy depicts the correct configuration of the circuit. Next, the circuit accuracy and runtime are computed for every VLSI models.

\section{IMPLEMENT ATION}

For implementation, firstly, we generate random program k-SAT clauses. From there, we in itialized initial states for the neurons in the clauses. The network will evolve until final state reached. Once the program has reached the final state, the neuron state is updated via equation (9). As soon as the network relaxed to an equilibrium state, test the final state obtained for the relaxed neuron whether it is a stable state. Stable state will be considered provided the state remains unchanged for five runs. According to Pin kas [18], letting an ANN to evolve eventually shall lead to stable state where the energy function obtained does not change further. In this case, the corresponding final energy for the stable state will be calculated. If the difference between the final energy and the global minimum energy is within the given tolerance value, then consider the solution as global solution.

\section{RESULTS AND DISCUSSION}

The performances of our proposed paradig $m$ have been evaluated based on global VLSI configuration, circuit accuracy and the runtime.

\section{A. Global VLSI configuration}

Table 1. Global VLSI configuration for HNN-2SAT circuit

\begin{tabular}{|c|c|}
\hline Number of Transistors & The Correct VLSI Models \\
\hline 20 & 9976 \\
\hline 40 & 9825 \\
\hline 60 & 9756 \\
\hline 80 & 9787 \\
\hline 100 & 9524 \\
\hline 120 & 9132 \\
\hline
\end{tabular}

Table 2. Global VLSI configuration for HNN-3SAT circuit

\begin{tabular}{|c|c|}
\hline Number of Transistors & The Correct VLSI Models \\
\hline 20 & 9980 \\
\hline 40 & 9901 \\
\hline 60 & 9826 \\
\hline 80 & 9698 \\
\hline 100 & 9586 \\
\hline 120 & 9233 \\
\hline
\end{tabular}

Table 1 and 2 elucidate the global VLSI configuration for different number of transistors. The result of the transistor HNN-2SAT and HNN-3SAT problem are summarised in table 1 and table 2 respectively. In this study, we are successfully reconstruct the VLSI models via computer simulations. The ability for the proposed algorithm can be analysed by looking at the number of correct VLSI models for higher number of transistors. The proposed algorithm had retrieved the correct transistor model for the circuit. It can be deduced that, almost $90 \%$ of the HNN-2SAT and HNN-3SAT model having $5 \mathrm{~V}$ output. As the number of transistor increased, the retrieval power of HNN-2SAT and HNN-3SAT 
decreased. This is due to spurious effect of the network that cause the network to stuck at sub-optimal minima. As an outcome, HNN-2SAT and HNN-3SAT recalled a wrong model for the transistor state. The concept are similar to the global minima solutions in the neural network done by Sathasivam [3, 24]. As the number of neurons increased, the global minima ratio will decreased. Same goes to our proposed algorith $\mathrm{m}$. When the VLSI models get more complex (higher number of transistors), the number of correct or functional circu it will decreased. It is obvious that the number of correct VLSI models are reduced significantly when the number of transistor increased.

\section{B. Circuit Accuracy}

In this study, circuit accuracy is computed based on the percentage of correctness for the recalled VLSI models from the Hopfield's Content Addressable Memory (CAM).

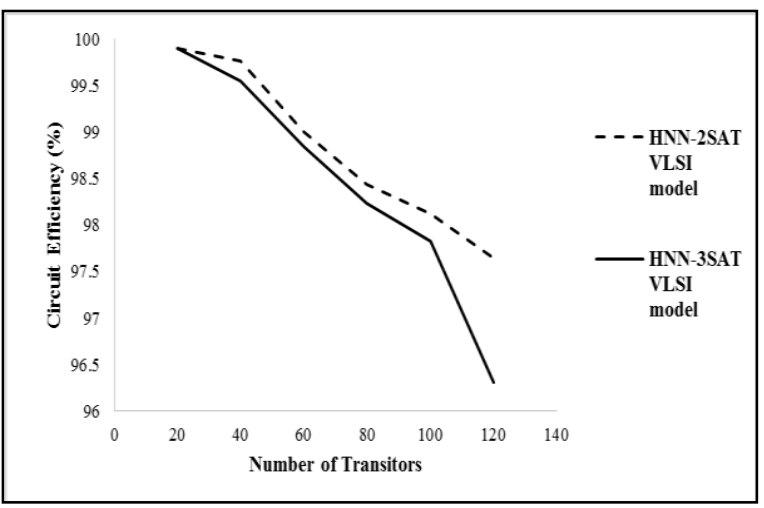

Fig.4. Circuit Efficiency for HNN-2SAT and HNN-3SAT VLSI model

Figure 4 depicts that HNN-2SAT and HNN-3SAT are able to retrieve greater than $95 \%$ correct model. Hence, the proposed algorithms can be used in reconstructing more complex VLSI models. This is because the retrieval power for both models are slightly consistent even though the number of transistors increased. The correctness of the output will be almost $100 \%$. Relatively high circuit efficiency shows that, HNN-2SAT and HNN-3SAT has a greater stability when more transistors are introduced. In addition, better efficiency will improve the functionality of normal VLSI circuit as the complexity increases. The efficient learning algorithm will help to reduce the complexity of the algorithm. In our study, it can be clearly seen that HNN-2SAT and HNN-3SAT can be implemented as complex VLSI models. Thus, the circuit mis s rate can be minimized.

\section{Circuit Runtime}

Circuit runtime is another indicator to check the effectiveness of VLSI models. The performance of VLSI models can be determined via the time taken for the circuit to fully functional. Theoretically, as the number of transistors in VLSI model increases, the circuit runtime will increases.
Table 3. Circuit Runtime for HNN-2SAT and HNN-3SAT VLSI models

\begin{tabular}{|c|c|c|}
\hline $\begin{array}{c}\text { Number of } \\
\text { Transistors }\end{array}$ & $\begin{array}{c}\text { HNN-2SAT } \\
\text { VLSI Models } \\
\text { (seconds) }\end{array}$ & $\begin{array}{c}\text { HNN-3SAT } \\
\text { VLSI Models } \\
\text { (seconds) }\end{array}$ \\
\hline 20 & 10 & 8 \\
\hline 40 & 33 & 24 \\
\hline 60 & 85 & 69 \\
\hline 80 & 205 & 188 \\
\hline 100 & 2345 & 2002 \\
\hline 120 & 9880 & 7655 \\
\hline
\end{tabular}

Table 3 depicts the runtime of the circuit with different number of transistors for HNN-2SAT AND HNN-3SAT VLSI model. It can be seen clearly that, if the number of transistor or switch is lesser, the circuit runtime is basically faster. This is due to the less burden to generate the output correspond to $5 \mathrm{~V}$ for each of clauses or transistors. In other words, the complexity of the circuit affects the runtime for the VLSI models. The concept is related to the neurons in Hopfield logic programming. The complexity of the network increased as the network gets enormous. Hence, this is because of a condition when the network getting larger and complex, the network is likely to get stuck in local minima and consume more computation time. As a result, more runtime is needed to relax to global solution as the number of neurons increased. The neurons need to jump massive energy barrier to reach the global solutions.

According to our HNN-2SAT and HNN-3SAT VLSI circuit, we observed that the runtime for the circuit is directly proportional to the nu mber of transistor. This key active electronic component is vital to ensure the circuit to be operated properly. Most of the runtime spent during the process of producing $5 \mathrm{~V}$ output in each clauses for every VLSI models. If the transistor combination doesn't satisfied, the final output will be $0 \mathrm{~V}$ and the overall VLSI model will function improperly. In our study, if the number of these active electronic components are exceeding 120, the circuit need more runtime before it can be declared functional. All in all, complex circuit will consume time in order to complete the operation or in our cases to be satisfied. Better optimization technique can be applied to improve the circuit runtime for highly complex VLSI models.

\section{CONCLUSION}

We have presented our proposed algorithms, namely HNN-2SAT and HNN-3SAT VLSI models to check the VLSI circuit correctness if the number of transistors gets higher. It had been shown by the computer simulations our proposed models that incorporated with Hopfield neural networks were able to retrieve the desired output as the traditional VLSI models did. Hence, the proposed models are supported by the very good agreement of global VLSI configuration, circuit accuracy and circuit runtime obtained. Thus, our models can be integrated to solve more complicated electronics problem. 


\section{REFERENCES}

[1] S. Sathasivam, Learning in the Recurrent Hopfield Network, Proceedings of the Fifth International Conference on Computer Graphics, Imaging and Visualisation, pp. 323-328, 2008.

[2] W.A.T. Wan Abdullah, Logic Programming on a Neural Network. Malaysian Journal of computer Science, 9 (1), pp. 1-5, 1993.

[3] S. Sathasivam, Energy Relaxation for Hopfield Network with the New Learning Rule, International Conference on Power Control and Optimization, pp. 1-5, 2009.

[4] M. Velavan, Boltzman Machine and Hyperbolic Activation Function in Higher Order Network, 9 (2), pp. 140-146, 2014.

[5] S. Sathasivam, P.F. Ng, N. Hamadneh, Developing agent based modelling for reverse analysis method, 6 (22), pp. 4281-4288, 2013.

[6] J. F. Hurdle, E. L. Brunvand, L. Josephson, Asynchronous VLSI design for Neural system implementation, VLSI for Neural Networks and Artificial Intelligence, pp. 129-139, 1994.

[7] G. Gopalakrishnan, L. Josephson, Towards Amalgamating the Synchronous and Asynchronous Styles, Proc. TAU 93: Timing Aspect of VLSI, 1993.

[8] R. Puff, J. Gu, A BDD SAT solver for satisfiability testing: An industrial case study, Annals of Mathematics and Artificial Intelligence, 17 (2), pp. 315-337, 1996.

[9] F. A. Aloul, A. Sagahyroon, Using SAT-Based Techniques in Test Vectors Generation, Journal of Advance in Information Technology, 1 (4), pp. 153-162, 2010.

[10] J. R. burch, E. M. Clarke, D. E. Long, L. McMillan, D. L. Dill, " Sequential Circuit Verification Using Symbolic Model Checking," Proceedings of the $27^{\text {th }}$ ACM/IEEE Design Automation Conference, pp. 46-51, 1990.

[11] J. R. Burch, E. M. Clarke, D. E. Long, L. McMillan, D. L. Dill, Symbolic model checking: 1020 states and beyond, Logic in Computer Science, LICS'90, Proceedings of the $5^{\text {th }}$ Annual IEEE Symposium, pp. 428-439, 1990.

[12] K. J. Lang, Hill Climbing Beats Genetic Search on a Boolean Circuit Synthesis Problem of Koza's, Proceedings of the $12^{\text {th }}$ International Conference on Machine Learning, pp. 340-343, 2014.

[13] H. S. Jin, H. J. Han, F. Somenzi, Efficient conflict analysis for finding all satisfying assignments of a boolean circuit, in Tools and Algorithms for the Construction and Analys is of Systems, Berlin, Heidelberg: Springer, pp. 287-300, 2005.

[14] T. A. Junttila, I. Niemela, Towards an efficient tableau method for Boolean circuit satisfiability checking, in Computational Logic-CL 2000, Berlin, Heidelberg: Springer, pp. 553-567, 2000.

[15] A. Cimatti, M. Roveri, Bertoli. P, Conformant planning via symbolic model checking and heuristic search, Artificial Intelligence, 159 (1), pp. 127-206, 2004.

[16] M. Formann, F. Wagner, The VLSI layout problem in various embedding models, Graph-Theortic Concepts in Computer Science, Berlin, Heidelberg: Springer, pp. 130139, 1990.

[17] J. J. Hopfield, D. W. Tank, Neural computation of decisions in optimization problem, Biological Cybernatics, 52, pp. 141-152, 1985.

[18] G. Pinkas, R. Dechter, Improving energy connectionist energy minimization, Journal of Artificial Intelligence Research, 3, pp. 223-15, 1995.
[19] D. Vilhelm, J. Peter, \& W. Magnus, Counting models for 2SAT and 3SAT formulae. Theoretical Computer Science, 332 (1), pp. 265-291, 2005.

[20] R. Rojas, Neural Networks: A Systematic Introduction. Berlin: Springer, 1996.

[21] N. Siddique, H. Adeli, Computational Intelligence Synergies of Fuzzy Logic, Neural Network and Evolutionary Computing. United Kingdom: John Wiley and Sons, 2013.

[22] R.A. Kowalski, Logic for Problem Solving. New York: Elsevier Science Publishing, 1979.

[23] B. Sebastian, H. Pascal and H. Steffen, Connectionist model generation: A first-order approach, Neurocomputing, 71(13), pp. 2420-2432, 2008.

[24] S. Sathasivam, Upgrading Logic Programming in Hopfield Network, Sains Malaysiana, 39, pp. 115-118, 2010.

[25] B. Tobias and K. Walter, An improved deterministic local search algorithm for 3-SAT, Theoretical Computer Science 329, pp. 303-313, 2004.

[26] U. Aiman and N. Asrar, Genetic algorithm based solution to SAT-3 problem, Journal of Computer Sciences and Applications, 3, pp. 33-39, 2015.

[27] S. Haykin, Neural Networks: A Comprehensive Foundation, New York: Macmillan College Publishing, 1999.

[28] J. Gu, Local Search for Satisfiability (SAT) Problem, IEEE Transactions on Systems, Man and Cybernetics, vol. 23 pp. 1108-1129, 1993.

[29] W. Fernandez, Random 2-SAT: Result and Problems, Theoretical Computer Science, 265, pp. 131-146, 2001.

[30] C. Meadm, L. Conway, Introduction to VLSI system, Reading, MA: Addison-Wesley, 1980.

[31] R. R. Schaller, Moore's law: past, present and future. Spectrum, 34(6), pp. 52-59, 1997.

[32] R. Sethuram and M. Parashar, Ant colony optimization and its application to Boolean satisfiability for digital VLSI circuits, Advanced Computing and Communications, IEEE, pp. 507-512, 2006.

[33] A. D. Pwasong and S. Sathasivam, Forecasting Performance of Random Walk with Drift and Feed Forward Neural Network Models, International Journal of Intelligent System and Application, vol. 23 pp. 49-56, 2015.

[34] P. Whig and S.N. Ahmad, Performance analysis of various readout circuits for monitoring quality of water using analog integrated circuits, International Journal of Intelligent Systems and Applications, 4 (11), pp. 91-98, 2012.

[35] S. Koussoube, R. Noussi and B.O. Konfe, Using Description Logics to specify a Document Synthesis System. International Journal of Intelligent Systems and Applications, 5(3), p.13, 2013.

\section{Authors' Profiles}

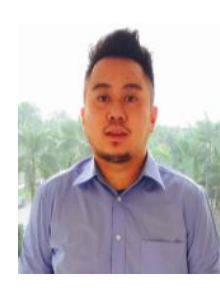

Mohd Asyraf Mansor was born in Sarawak, Malaysia in 1990. He obtained his MSc (2014) and $\mathrm{BSc}(\mathrm{Ed})$ (2013) from Universiti Sains Malaysia. He is currently pursuing Ph.D degree at School of Mathematical Science, Universiti Sains Malaysia. His current research interests include evolutionary algorithm, satisfiability problem, neural networks, logic programming and heuristic method. 


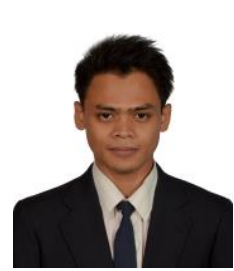

Mohd Shareduwan bin M. Kasihmuddin received his MSc (2014) and BSc(Ed) (2013) from Universiti Sains Malaysia. $\mathrm{He}$ is currently pursuing Ph.D degree with School of Mathematical Science, Universiti Sains Malaysia Penang Malaysia. His current research interests include neuro-heuristic method, constrained optimization, neural network and logic programming.

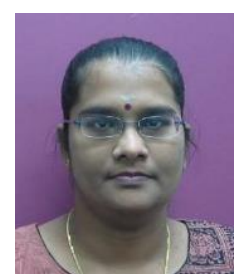

Saratha Sathasivam is a lecturer in the School of Mathematical Sciences, Universiti Sains Malaysia. She received her $\mathrm{MSc}$ and $\mathrm{BSc}(\mathrm{Ed})$ from Universiti Sains Malaysia. She received her Ph.D at Universiti Malaya, Malaysia. Her current research interest are neural networks, agent optimization problem. based modeling and constrained

How to cite this paper: Mohd Asyraf Mansor, Mohd Shareduwan M. Kasihmuddin, Saratha Sathasivam, "VLSI Circuit Configuration Using Satisfiability Logic in Hopfield Network", International Journal of Intelligent Systems and Applications (IJISA), Vol.8, No.9, pp. 22-29, 2016. DOI: 10.5815/ijisa.2016.09.03 